\title{
Mate quality bias: Sex differences in humans
}

\author{
Antonios Vakirtzis* \& S. Craig Roberts
}

School of Biological Sciences, Crown St, University of Liverpool, L69 7BX, Liverpool, UK (corresponding author's e-mail: a.vakirtzis@liverpool.ac.uk)

Received 8 June 2009, revised version received 3 Nov. 2009, accepted 18 Sep. 2009

Vakirtzis, A. \& Roberts, S. C. 2010: Mate quality bias: Sex differences in humans. - Ann. Zool. Fennici 47: 149-157.

In mate choice copying, a male is more likely to be chosen by other females simply by being observed mating. A recent finding is that women are influenced in their assessments of men by the phenotypic quality of males' sexual partners. We recently proposed that the term 'mate quality bias' should be used to differentiate this phenomenon from 'mate choice copying'. Here, under the guise of a dating preferences survey we replicated and extended some earlier results. We found that when presented to female raters, men are more desirable dates when they are depicted as having had relatively attractive (versus relatively unattractive) former partners, an effect that appears to be moderated by a second variable, namely the former partner's age. We did not find evidence for this bias effect when men rate women whose profiles have been similarly manipulated. These findings suggest the operation of a sex-specific mate choice mechanism.

\section{Introduction}

Traditionally, scientists have viewed mate choice as though undertaken by individuals that are not influenced by the mating decisions of others. Recently, however, a growing literature has supported the idea that individual females are often influenced in their choices by the actions of other females (Pruett-Jones 1992, Dugatkin 1996, Galef \& White 2000, Westneat et al. 2000, White 2004). In mate choice copying (Losey et al. 1986), a female adopts the choices of other females, mating with previously mated males or rejecting previously rejected males (Dugatkin 1992, Witte \& Ueding 2003). Copying is a strategy that can allow females to minimize the sampling costs associated with a process of active mate choice (Pruett-Jones 1992), which include the time and energy that must be spent inspecting potential mates, the associated predation risks and harassment by rejected males (Reynolds \& Gross 1990, Gibson \& Höglund 1992, Andersson 1994). Copying may also evolve for other reasons, such as enhancing female discrimination accuracy (Gibson \& Höglund 1992, Nordell $\&$ Valone 1998). For instance young and sexually inexperienced females often have inferior discriminatory abilities to the rest of the female population. This creates an informational asymmetry: while these females have a lot to gain from copying the mating decisions of more able females, the reverse does not hold (Nordell \& Valone 1998). This has been empirically confirmed in the guppy, Poecilia reticulata, where it was shown that while young females copy the decisions of their older conspecifics, the latter are not influenced by the mating decisions of the former (Amlacher \& Dugatkin 2005, Dugatkin 
\& Godin 1993). Copying has now been documented in a variety of fish and bird species (e.g. Dugatkin \& Godin 1992, Hoglund et al. 1995, Galef \& White 1998, Dugatkin et al. 2003, Witte \& Massmann 2003, Munger et al. 2004, Widemo 2006).

In a highly social species like humans, information about sexual relationships is often readily available to third parties, either through direct observation or gossip. Uller and Johansson (2003) examined the validity of the socalled 'wedding ring effect' (Knight 2000), the folk notion that women find married men more attractive than their single peers. Two male assistants interacted with female raters while either wearing or not wearing a wedding ring. Contrary to the researchers' expectations, the men were rated as less desirable mates on a variety of measures when wearing the ring than without it, a result that was the opposite of what a mate-choice copying hypothesis would suggest. Another study with a Finnish sample using photographic stimuli of men presented with and without girlfriends (the exact term used was naisystävä, which denotes a sexual relationship but without regular dating) also found no evidence of mate choice copying (Milonoff et al. 2007). A study in Canada, using pictures of men accompanied by a brief description of the men and their interests, produced positive results (Eva \& Wood 2006). Here the independent variable was the assignation of described marital status, with half the female raters seeing the men as 'Married' and the other half as 'Single'. The women who saw the men in their 'Married' condition rated them as significantly more attractive. Finally, a study with a British sample found that the attractiveness ratings a man received when presented alone did not, on average, differ from those he received when presented with the picture of his supposed girlfriend (Waynforth 2007).

What can explain these mostly negative results is the different mating system of humans as compared with that of the other species where copying has been researched (Uller \& Johansson 2003, Milonoff et al. 2007, Vakirtzis \& Roberts 2009). The idea of mate-choice copying arose originally through observations of the highly skewed distributions of male mating success in leks (Bradbury \& Gibson 1983, Losey et al.
1986, Wade \& Pruett-Jones 1990). In lekking species a minority of males secure the vast majority of matings, with a very large proportion of males remaining unmated (e.g. Bradbury \& Gibson 1983, Alatalo et al. 1992). Male choice is minimal, and male sexual behavior is organized around the single goal of maximizing the number of sexual partners and frequency of copulations. There is therefore presumably a direct and unambiguous relation between the number of sexual partners a male secures and his quality.

The human mating system is completely different. It is characterized, among other things, by relatively low variance in quantitative male mating success, strong male choice, considerable paternal investment and a less clear-cut relationship between number of sexual partners and male quality. Data from the United Nations demographic yearbooks show that, cross-culturally, nearly $92 \%$ of men succeed in marrying by the age of 49 (as cited in Fisher 1989). An examination of non-marital sexual relations in the United States, for which there is an abundance of data, reveals a similar picture. In the age range of 25-59 years, $91 \%$ of men participating in the 1988-1990 General Social Surveys report being sexually active in the preceding year (as cited in Seidman \& Reider 1994). Of these, 80\% report having had one sexual partner in the preceding year, with another $12 \%$ reporting two or three partners (Seidman \& Rieder 1994). In a setting like this, where the vast majority of adult males are sexually involved with one female at a time, mating status (single $v s$. partnered) per se is a cue with very little value in mate choice, since there is not enough variance in this cue to allow for efficient discrimination (Vakirtzis \& Roberts 2009). Adding to this, the relationship between number of partners and male quality is not as clear-cut as it is in lekking or polygynous species. For example married men and men with more education tend to have fewer, rather than more, lifetime sexual partners (Billy et al. 1993), but it could not be seriously argued that these are the categories of men most likely to contain individuals of lower mate value.

Taken together, the evidence we have discussed suggests that women almost certainly don't engage in mate-choice copying as females of other species do. An idea that is becoming 
increasingly popular in recent years is that, rather than being sensitive merely to if a man has a sexual partner or not, women could extract more information about a prospective mate by evaluating his current or previous partners' mate value, as revealed through her attractiveness (Uller \& Johansson 2003, Waynforth 2007, Vakirtzis \& Roberts 2009). We recently suggested that the term 'mate quality bias should' be used to describe this phenomenon, for its evolutionary dynamics clearly set it apart from mate choice copying (Vakirtzis \& Roberts 2009). We also suggested that mate quality bias will usually be found in mating systems characterized by mutual choice and serial monogamy, as in humans (for discussion see Vakirtzis \& Roberts 2009).

Recently, Waynforth (2007) has produced the first experimental evidence that women are affected by the attractiveness of mens' sexual partners. He found that an image of a man presented next to an attractive woman (supposedly his girlfriend) elicited higher attractiveness ratings from female raters as compared with an initial rating of the same male image presented alone, and that the size of the change in rating was mainly determined by the attractiveness of the supposed girlfriend. It is especially worth noting that men presented with relatively unattractive girlfriends actually received lower attractiveness ratings to those they received when presented on their own (Waynforth 2007). Similar findings were reported by Little et al. (2008), namely that when rating images of prospective long-term partners, women prefer men presented next to feminine rather than masculine female faces (supposedly their girlfriends), and men prefer women presented with masculine rather than feminine male faces (supposedly their boyfriends). Feminine female faces and masculine male faces are generally also found to be more attractive (Little et al. 2008).

Here, we try to replicate these general findings and test for gender differences in mate quality bias by using a slightly different procedure. The experiment was designed in a way that minimized the danger of the raters simply being coaxed into adjusting their ratings of the prospective mates according to the images of the partners, as would perhaps occur if the two images were simply presented simultaneously without any other explanation (as they were in both experiments where the effect was found, Waynforth 2007, Little et al. 2008). The goal was to mask the purpose of the experiment so as to ensure that any reaction to the independent variable would manifest spontaneously (as in Uller \& Johansson 2003, Eva \& Wood 2006, Milonoff et al. 2007). For this reason the image of the former partner was merely one among several sources of information. We presented sets of male facial images to female raters. These images were accompanied by a fictional testimony from a former partner and a female facial image of this supposed partner. Each male profile (image and testimony) was presented in two conditions, with either an unattractive or an attractive former partner; it is important to note that the testimony did not change across conditions and cannot therefore be responsible for any effect we show in relation to the attractiveness of former partners. Each female rater viewed the male in only one condition. We predicted that an attractive ex-partner would increase the male's attractiveness rating. We also tested whether male judgments of females would be influenced by attractive and unattractive ex-partners in the same way.

\section{Material and methods}

\section{Subjects}

A total of 40 female students (mean age $\pm \mathrm{SD}=$ $21.4 \pm 2.2$ years $)$ and 40 male students $(20.3 \pm$ 1.8 years) were recruited by convenience from around the University of Liverpool campus.

\section{Material and design}

To create the stimulus sets, we used 170 female (mean age $\pm \mathrm{SD}$ at time of photo $=20.9 \pm 1.9$ years) and 128 male $(20.9 \pm 2.9)$ color facial photos of young adults who had participated in previous mate choice studies (Roberts et al. 2005a, 2005b, 2008). These participants were students and staff from the University of Newcastle, and thus were unfamiliar to our experimental participants at Liverpool. Using a java applet to 
present images in randomized order, seven male judges (students at the University of Liverpool) viewed all 170 female images (one at a time) on a computer screen, and by pressing the appropriate key on the keyboard rated each one for attractiveness on a Likert-type scale from 1 (least attractive) to 5 (most attractive). Ratings from the seven judges were then averaged to produce a single attractiveness rating to each female photo. In the same manner, attractiveness ratings from seven female judges (also students at the University of Liverpool) were averaged into a single attractiveness rating for each male photo.

\section{Stimuli used with female raters}

To obtain comparable sets of attractive and unattractive images, which were of a sufficiently different level of attractiveness while avoiding individuals lying at the extremes of the attractiveness score distribution, we selected the 10 images either side of the 25th and 75th percentiles (20 "unattractive" and 20 "attractive" images) as the experimental stimuli. From the male images, we selected 20 images of approximately average attractiveness (10 images either side of the median). In cases where attractiveness scores were tied for images at the cutoff points, we randomly picked the necessary number of images to include in the stimulus set. For each male image, we randomly assigned two female images, one from the "attractive" and one from the "unattractive" sets.

Fictitious profiles were then created for the 20 male photos. These consisted of a name, subject of studies and brief comments (between one and three) by an ex girlfriend. Some comments were personality descriptions without reference to the relationship (e.g. 'He is sweet and caring'). Others made direct reference to the relationship (e.g. 'I just found our relationship unexciting sometimes' or 'Sometimes he would get jealous for no apparent reason'). Although most profiles included both positive and negative comments, for the sake of realism some consisted of only negative or positive comments. A profile was then randomly assigned to each of the 20 males.

Using this material we created 40 distinct computer slides, two for each male stimulus.
Each slide featured a large, centrally placed image of the male photo (approx. $13 \times 9.6 \mathrm{~cm}$ ), beneath which were written his name and subject of studies. Immediately beneath these were the brief comments, encircled by a speech bubble that originated from the smaller photo of the exgirlfriend (approx. $8 \times 6 \mathrm{~cm}$ ), which was at the lower left corner of the screen. The design of the experiment was within-stimulus, with the two slides for each male stimulus $(N=20)$ differing only with respect to the image of the ex-girlfriend that supposedly provided the comments. In all other respects (name, subject of studies and testimony) the two slides were identical.

\section{Stimuli used with male raters}

Selection of stimuli was carried out in similar fashion as for female raters. Briefly, we selected 20 female images from the 170 available (10 either side of the median attractiveness score) in order to have a set of females of approximately average attractiveness. From the male images, we selected 10 images either side of the 25 th and 75th percentiles (20 "unattractive" and 20 "attractive" men). Two male images, one relatively attractive and one relatively unattractive, were randomly assigned to each female image. The 20 fictitious profiles previously used to describe men were now modified in order to describe women (i.e. mostly altering personal pronouns to reflect sex appropriately, from "he" to "she", "his" to "hers", and so on). Each female image was randomly assigned a profile. Using this material we again created 40 distinct computer slides, two for each female stimulus in the manner described above. Again the experiment was within-stimulus, with the two slides for each female stimulus $(N=20)$, differing only with respect to the image of the ex-boyfriend that supposedly provided the comments.

\section{Procedure}

Subjects were told they would be participating in a dating preferences survey. No payment was given. The procedure was computer-based using a PowerPoint presentation, and apart from the 
instructions on the screen at the beginning of the test, no other instructions were offered. The instruction screen informed the subjects that they would be shown a small sample of oppositesex students from nearby universities, together with personality descriptions from a partner with whom 'they were romantically involved in the past, for a minimum of three months'.

Each female rater viewed the 20 male stimuli in random order, and in a random mix of "attractive ex" and "unattractive ex" conditions. Ratings were recorded on paper. For every male stimulus two dependent variables, 'overall attractiveness' and 'willingness to go on a date' were scored on a scale of one to seven. Overall, each male stimulus was viewed 20 times in each condition (attractive and unattractive ex). The same procedure was used for the male raters who viewed the 20 female stimuli. Participants were debriefed at the end of the procedure.

\section{Results}

Each participant's ratings, for both dependent variables, were standardized prior to analysis to control for individual differences in use of rating scales. All tests were two-tailed.

\section{Men rating women shown with ex-boyfriends}

There was no difference in rating between conditions (attractive versus unattractive ex-boyfriends) for either overall attractiveness (Wilcoxon signed-rank test: $Z=-0.149, N=20, P=$ 0.881 ) or willingness to date (Wilcoxon signedrank test: $Z=-0.672, N=20, P=0.502)$. These results show that men were clearly not influenced in their judgments by the attractiveness of womens' supposed ex-boyfriends.

\section{Women rating men shown with ex-girlfriends}

There was a tendency for men to receive higher ratings in their 'attractive ex' condition for 'overall attractiveness', though this difference did

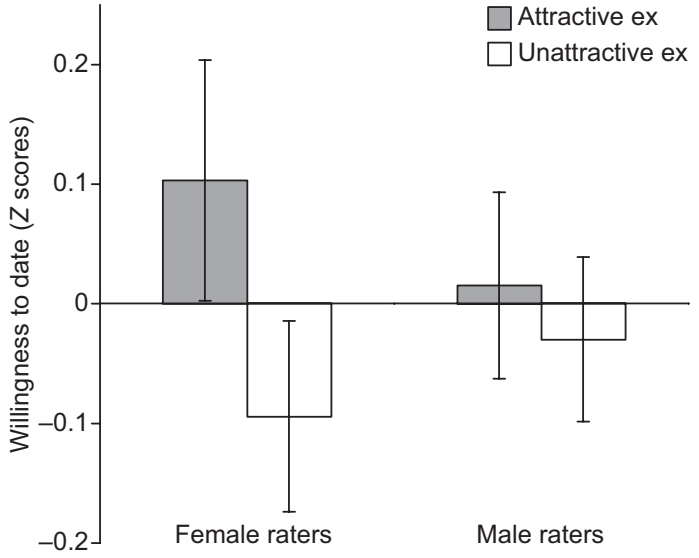

Fig. 1. Differential sensitivity of women and men to the attractiveness of a prospective mate's former partner. While women indicate significantly greater willingness to date individuals previously in a relationship with a relatively attractive (compared to relatively unattractive) partner, this is not the case for men. Data are means and standard errors.

not achieve statistical significance (Wilcoxon signed-rank test: $Z=-1.79, N=20, P=0.071$ ). The difference for 'willingness to date' was significant and in the same direction, with men in the 'attractive ex' condition being rated as more desirable dates (Wilcoxon signed rank test: $Z=$ $-2.44, N=20, P=0.014)$. The results for female raters were thus markedly different to those obtained for male raters (Fig. 1).

It had been anticipated that 'willingness to date' would be the dependent variable most likely to show an interaction with the independent variable (see Discussion). This result was in the predicted direction, yet the fact that in five of 20 cases men received higher mean scores when presented in the 'unattractive ex' condition led us to conduct a post-hoc examination of the data for possible confounding variables. We first noticed that in all five of these counter-instances the female in the 'unattractive-ex' condition was older at the time the photograph had been taken than her paired female in the 'attractive ex' condition. This led us to find that the signed age difference in years between each male's two exgirlfriends (attractive minus unattractive) correlated positively with the signed difference (attractive minus unattractive) in average 'willingness to date' (Spearman rank correlation: $r_{\mathrm{s}}=0.521$, 


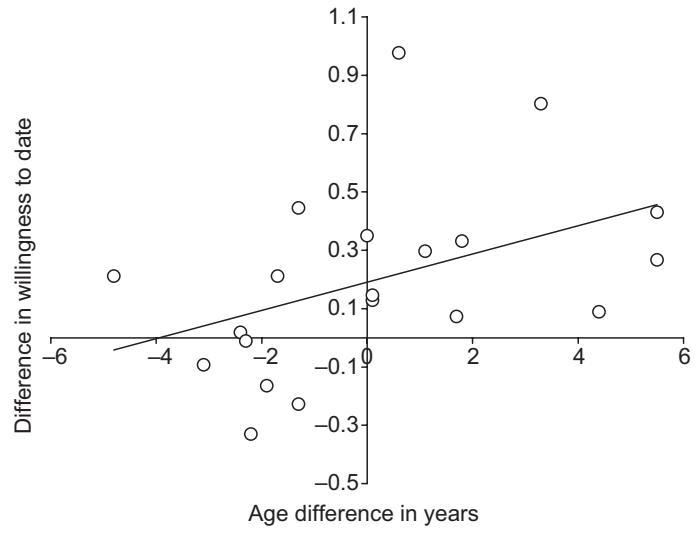

Fig. 2. Relationship between age difference of attractive and unattractive female ex-partners and men's desirability as a date. The difference between mean 'willingness to date' scores attributed to each male ( $N$ $=20$ ) in the two conditions ('attractive ex' minus 'unattractive ex') is plotted on the vertical axis. The age difference in years between each male's ex-girlfriends (attractive minus unattractive) is plotted on the horizontal axis. The two variables correlate positively $(P=$ 0.018).

$N=20, P=0.018$; Fig. 2). This result suggests that the women participants were influenced in their ratings not only by the attractiveness of the females in the photos but also by their age, a variable which must necessarily correlate positively with sexual experience. Although the females had initially been paired solely on the basis of attractiveness, the age distribution (mean $\pm \mathrm{SD}=21.2$ \pm 2.5 years) of the relatively attractive set did not differ from that $(21.1 \pm 2.0$ years $)$ of the unattractive set (Mann-Whitney $U$ test: $U=193, N_{1}=20$, $N_{2}=20, P=0.850$ ), so the primary attractiveness effect we found could not be attributed to chance age differences between the attractive and unattractive sets of ex-girlfriends.

\section{Discussion}

Our results confirm that men increase their value in the mating market by dating attractive women (Waynforth 2007). We found that women are sensitive to the facial attractiveness of prospective mates' previous sexual partners. Men were rated as more desirable dates when perceived to have been formerly involved with relatively attractive women. This effect appeared to be confounded by a second variable, namely the age of the former partner. We found indirect evidence for a positive relationship between the age of a young woman with whom a man had been supposedly involved and his subsequent desirability as a date. This finding is in line with the expectation that women should assign greater significance to the choices of more experienced females and lesser significance to those of relatively inexperienced females (Nordell \& Valone 1998). Similar results have been obtained for the guppy (Amlacher \& Dugatkin 2005, Dugatkin $\&$ Godin 1993), so this reliance on the choices of relatively older, more experienced females is probably a common evolutionary pressure wherever nonindependent mate choice operates. It should, however, be stressed that the female stimuli in this study were all young adults (mean age $20.9 \pm 1.9$ years), and there is obviously an age limit to this effect, as after a certain age the mate value of a woman declines. But even in these young ages there is undoubtedly considerable difference in experience between, say, an 18-year old and 23-year old woman. It is interesting to note that Waynforth (2007) found an analogous pattern from the point of view of the rater, with women raters who reported more lifetime sex partners showing decreased bias behavior, a finding that can be attributed to these women's greater experience in mate choice. Our study highlights the potential fruitfulness of focusing future study on the specific cues that women use when biasing their reactions to men on the basis of their partners' appearance. The primary cue is almost certainly the partner's attractiveness, but cues relating to age (as found here), confidence, sexual experience or personality could also be picked up by other women.

In contrast to a recent study (Little et al. 2008), we did not find a comparable pattern when males assessed females presented with their ex-partners. This discrepancy could be due to a number of factors, such as our use of real faces compared with Little et al.'s (2008) use of composite faces, or the fact that the males shown here were supposedly former partners rather than supposedly current partners. It should also be noted that in Little et al.'s (2008) study, the independent variable was not partner attractiveness per se, but rather partner masculinity/femininity. 
Perhaps more significantly, there were important differences in the way in which images were displayed in the two studies. Whereas Little et al. (2008) presented male raters with no stimuli other than two faces of equal salience on the screen, we tried to present the partner's image as inconspicuously as possible, together with other sources of information that justified the presence of the partner's image and attempted to increase ecological validity and obscure the real purpose of the task. Future research is needed to determine which of these factors could explain these differences in results.

The gender specificity in relation to mate quality bias that we find probably relates to sex differences in the mate choice criteria used by the two sexes. The mate value of women is determined primarily by physical cues of nubility and attractiveness, whereas the mate value of men depends more on non-physical characteristics like social status and resource holding potential (reviewed in Buss 1994, 1999, Roberts \& Little 2008), variables which are considerably more difficult to evaluate. A female with limited knowledge of the local mating market can rapidly improve her estimates of males' relative quality by being sensitive to the attractiveness of their sexual partners, a readily available visual cue that can be assessed instantly (Waynforth 2007). A number of studies have established a positive relation between womens' attractiveness and their husbands' income or occupational prestige, meaning that more attractive women tend to mate with more successful, high-status males (Elder 1969, Taylor \& Glenn 1976, Udry 1977, Udry \& Eckland 1984). It would be reasonable to assume that attractive women also tend to mate with males that rank highly on other desirable characteristics, such as ambition, industriousness and dependability (Buss 1999), which also cannot be reliably assessed very fast. In contrast, since as mentioned above physical cues are probably more prevalent in the decisionmaking process of males (Buss 1994, 1989), a male acting under similar conditions of uncertainty can acquire little additional information on a woman's mate value by assessing her male partner. A visual inspection of the female reliably captures many cues that play a significant role in the early stages of courtship, such as youthful appearance (Symons 1979, 1995), waist to hip ratio (Singh 1993, 2002) or facial attractiveness (Fink \& Penton-Voak 2002, Rhodes 2006).

We conclude with a hypothesis of how mate quality bias may operate in the real world. Mate choice in humans has been conceptualized as a series of decisions at various stages of the courtship process, with the aid of an ever-increasing amount of information (Miller \& Todd 1998). For example the first stage can consist of visual contact or the striking of a conversation, leading to the acquisition of more information about the prospective mate, which then determines whether or not the next stage (e.g. more socializing, leading to dating, etc.) will be pursued, or whether interest will be withdrawn and the whole affair terminated. Similar processes of sequential mating decisions have been found in other species (Backwell \& Passmore 1996, Gibson 1996). We suggest that a male's attractive mate could thus serve as an attention-grabbing stimulus for other females, increasing his chances of being noticed and securing, for instance, a first conversation or date. We find it unlikely that this early cue can have any persistent effect at later stages of the courtship process, especially in the face of unfavorable information obtained further down the line.

\section{Acknowledgments}

We thank Leena Lindström, Markus J. Rantala and an anonymous reviewer for comments and suggestions which improved the manuscript.

\section{References}

Alatalo, R. V., Höglund, J., Lundberg, A. \& Sutherland, W. J. 1992: Evolution of black grouse leks: female preferences benefit males in larger leks. - Behavioral Ecology 3: 53-59.

Amlacher, J. \& Dugatkin, L. A. 2005: Preference for older over younger models during mate-choice copying in young guppies. - Ethology, Ecology and Evolution 17: 161-169.

Andersson, M. 1994: Sexual selection. - Princeton University Press, Princeton (NJ).

Backwell, P. R. Y. \& Passmore, N. I. 1996: Time constraints and multiple choice criteria in the sampling behaviour and mate choice of the fiddler crab, Uca annulipes. Behavioral Ecology and Sociobiology 38: 407-416. 
Billy, J. O. G., Tanfer, K., Grady, W. R. \& Klepinger, D. H. 1993: The sexual behavior of men in the United States. - Family Planning Perspectives 25: 52-60.

Bradbury, J. W. \& Gibson, R. M. 1983: Leks and mate choice. - In: Bateson, P. (ed.) Mate choice: 109-138. Cambridge University Press, Cambridge.

Buss, D. M. 1989: Sex differences in human mate preferences: evolutionary hypotheses tested in 37 cultures. Behavioral and Brain Sciences 12: 1-49.

Buss, D. M. 1994: The evolution of desire: strategies of human mating. - Basic Books, New York.

Buss, D. M. 1999: Evolutionary psychology. - Allyn and Bacon, Boston.

Dugatkin, L. A. 1992: Sexual selection and imitation: females copy the mate choice of others. - The American Naturalist 139: 1384-1389.

Dugatkin, L. A. 1996: Copying and mate choice. - In: Heyes, C. M. \& Galef, B. G. (eds.), Social learning in animals: the roots of culture: 85-105. Academic Press, London.

Dugatkin, L. A. \& Godin, J. J. 1992: Reversal of female mate choice by copying in the guppy (Poecilia reticulata). - Proceedings of the Royal Society of London B 249: 179-184.

Dugatkin, L. A. \& Godin, J. J. 1993: Female mate copying in the guppy, Poecilia reticulata: age dependent effects. Behavioral Ecology 4: 289-292.

Dugatkin, L. A., Druen, M. W. \& Godin, J. J. 2003: The disruption hypothesis does not explain mate-choice copying in the guppy (Poecilia reticulata). - Ethology 109: 67-76.

Elder, G. H. 1969: Appearance and education in marriage mobility. - American Sociological Review 34: 519-533.

Eva, K. \& Wood, T. 2006: Are all the taken men good? An indirect examination of mate-choice copying in humans. - Canadian Medical Association Journal 175: 1573-1574.

Fink, B. \& Penton-Voak, I. 2002: Evolutionary psychology of facial attractiveness. - Current Directions in Psychological Science 11: 154-158.

Fisher, H. E. 1989: Evolution of human serial pairbonding. - American Journal of Physical Anthropology 78: 331-354.

Galef, B. G. \& White, D. J. 1998: Mate-choice copying in Japanese quail, Coturnix coturnix japonica. - Animal Behaviour 55: 545-552.

Galef, B. G. \& White, D. J. 2000: Evidence of social effects on mate choice in vertebrates. - Behavioural Processes 51: $167-175$.

Gibson, R. M. 1996: Female choice in sage grouse: the roles of attraction and active comparison. - Behavioral Ecology and Sociobiology 39: 55-59.

Gibson, R. M. \& Höglund, J. 1992: Copying and sexual selection. - Trends in Ecology and Evolution 7: 229-232.

Höglund, J., Alatalo, R. V., Gibson, R. M. \& Lundberg, A. 1995: Mate-choice copying in black grouse. - Animal Behaviour 49: 1627-1633.

Knight, J. 2000: Move over Casanova. - New Scientist 168: $30-33$.

Little, A. C., Burriss, R. P., Jones, B. C., DeBruine, L. M. \&
Caldwell, C. A. 2008: Social influence in human face preference: men and women are influenced more for long-term than short-term attractiveness decisions. Evolution and Human Behavior 29: 140-146.

Losey, G. S., Stanton, F. G., Telecky, T. M., Tyler, W. A. \& The Zoology 691 Graduate Seminar Class 1986: Copying others, an evolutionarily stable strategy for mate choice: a model. - The American Naturalist 128: 653-664.

Miller, G. F. \& Todd, P. M. 1998: Mate choice turns cognitive. - Trends in Cognitive Sciences 2: 190-198.

Milonoff, M., Nummi, P., Nummi, O. \& Pienmunne, E. 2007: Male friends, not female company, make a man more attractive. - Annales Zoologici Fennici 44: 348-354.

Munger, L., Cruz, A. \& Applebaum, S. 2004: Mate choice copying in female humpback limia (Limia nigrofasciata, family Poeciliidae). - Ethology 110: 563-573.

Nordell, S. E. \& Valone, T. J. 1998: Mate choice copying as public information. - Ecology Letters 1: 74-76.

Pruett-Jones, S. 1992: Independent versus nonindependent mate choice: Do females copy each other? - The American Naturalist 140: 1000-1009.

Reynolds, J. D. \& Gross, M. R. 1990: Costs and benefits of female mate choice: Is there a lek paradox? - The American Naturalist 136: 230-243.

Rhodes, G. 2006: The evolutionary psychology of facial beauty. - Annual Review of Psychology 57: 199-226.

Roberts, S. C. \& Little, A. C. 2008: Good genes, complementary genes and human mate choice. - Genetica 132: 309-321.

Roberts, S. C., Gosling, L. M., Carter, V. \& Petrie, M. 2008: MHC-correlated odour preferences in humans and the use of oral contraceptives. - Proceedings of the Royal Society of London B 275: 2715-2722.

Roberts, S. C., Little, A. C., Gosling, L. M., Jones, B. C., Perrett, D., Carter, V. \& Petrie, M. 2005a: MHC-assortative facial preferences in humans. - Biology Letters 1: 400-403.

Roberts, S. C., Little, A. C., Gosling, L. M., Perrett, D. I., Carter, V., Jones, B. C., Penton-Voak, I., Petrie, M. 2005b: MHC-heterozygosity and human facial attractiveness. - Evolution and Human Behavior 26: 213-226.

Seidman, S. N. \& Rieder, R. O. 1994: A review of sexual behavior in the United States. - The American Journal of Psychiatry 151: 330-340.

Singh, D. 1993: Adaptive significance of female physical attractiveness: Role of waist-to-hip ratio. - Journal of Personality and Social Psychology 65: 293-307.

Singh, D. 2002: Female mate value at a glance: Relationship of waist-to-hip ration to health, fecundity and attractiveness. - Neuroendocrinology Letters 23: 81-91.

Symons, D. 1979: The evolution of human sexuality. Oxford University Press, New York.

Symons, D. 1995: Beauty is in the adaptations of the beholder: the evolutionary psychology of human female sexual attractiveness. - In: Abramson, P. R. \& Pinkerton, S. D. (eds.), Sexual nature/Sexual culture: 80-118. The University of Chicago Press, Chicago.

Taylor, P. A. \& Glenn, N. D. 1976: The utility of education and attractiveness for females' status attainment through marriage. - American Sociological Review 41: 
484-498.

Udry, R. 1977: The importance of being beautiful: A reexamination and racial comparison. - American Journal of Sociology 83: 154-160.

Udry, R. \& Eckland, B. K. 1984: Benefits of being attractive: Differential payoffs for men and women. - Psychological Reports 54: 47-56.

Uller, T. \& Johansson, L. C. 2003: Human mate choice and the wedding ring effect: Are married men more attractive? - Human Nature 14: 267-276.

Vakirtzis, A. \& Roberts, S. C. 2009: Mate choice copying and mate quality bias: different processes, different species. - Behavioral Ecology 20: 908-911.

Wade, M. J. \& Pruett-Jones, S. G. 1990: Female copying increases the variance in male mating success. - Proceedings of the National Academy of Sciences of the
USA 87: 5749-5753.

Waynforth, D. 2007: Mate choice copying in humans. Human Nature 18: 264-271.

Westneat, D. F., Walters, A., McCarthy, T. M., Hatch, M. I. \& Hein, W. K. 2000: Alternative mechanisms of nonindependent mate choice. - Animal Behaviour 59: 467-476.

White, D. J. 2004: Influences of social learning on matechoice decisions. - Learning and Behavior 32: 105-113.

Widemo, M. S. 2006: Male but not female pipefish copy mate choice. - Behavioral Ecology 17: 255-259.

Witte, K. \& Massmann, R. 2003: Female sailfin mollies, Poecilia latipinna, remember males and copy the choice of others after 1 day. - Animal Behaviour 65: 1151-1159.

Witte, K. \& Ueding, K. 2003: Sailfin molly females (Poecilia latipinna) copy the rejection of a male. - Behavioral Ecology 14: 389-395. 